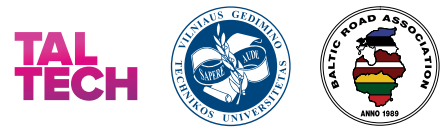

THE BALTIC JOURNAL

\title{
ELASTIC FOUNDATION \\ DISPLACEMENT APPROXIMATIONS
}

\begin{abstract}
VYTAUTAS KARGAUDAS ${ }^{1}$, NERIJUS ADAMUKAITIS ${ }^{2 *}$, MYKOLAS ŽMUIDA ${ }^{3}$,ALGIS PAKALNIS ${ }^{4}$, SAULIUS ZADLAUSKAS 5

1,2,3,5 Civil Engineering and Architecture Competences Centre, Kaunas University of Technology, Kaunas, Lithuania ${ }^{4}$ Dept of Transport Engineering, Kaunas University of Technology, Kaunas, Lithuania
\end{abstract}

Received 10 April 2018; accepted 07 September 2018

\begin{abstract}
Interaction of an elastic foundation and structures like beams, plates and frames plays an essential role in investigating soil media in contact and impact mechanics. The solution to this interaction problem is complicated even the foundation is assumed as a linear elastic medium. E. Winkler suggested the fair representation of the foundation in 1867, and then, to bring it closer to reality, an interaction between the spring elements was introduced. In this paper, a relatively simple membrane-spring system is investigated, where an ideal gas is added under or above the membrane. In many cases, this constant pressure in the cavity modifies the solution and accuracy of the approximation is significantly increased. The cases of concentrated normal force and uniform distributed load are examined. The results of elastic half-space line displacements and the membrane displacements are presented.
\end{abstract}

Keywords: additional pressure, continuum models, elastic foundation, spring membrane system, surface displacements.

\footnotetext{
* Corresponding author. E-mail: nerijus.adamukaitis@ktu.edu 


\section{Introduction}

The interaction between a foundation and supporting soil media is essential for structures and soil behaviour. The natural complexity of the problem has led to the development of many idealised models of soil, based on the classical theories of elasticity and plasticity (Vasani, 2003). Elastic models assume soil media as a linear elastic continuum. In Winkler (1867) model, surface displacement of the soil at every point is directly proportional to the stress applied. A similar model has also been used in the aerospace analysis to represent the effect of the air pressure of supersonic gas stream flow (Ashley, 1956). The real deflection at a surface point depends on the pressure at this point and also on the entire pressure distribution. The next grades are Filonenko-Borodich and Pasternak models. Initially, a generalisation of the Winkler model has been proposed introducing a shear layer at the top of the Winkler bed of linear springs (Filonenko-Borodich, 1940). This model is equivalent to dispose of a stretched wire at the top of the foundation columns (Pasternak, 1954). Several other kinds of extensions of the Winkler model are found in the specialised scientific literature to overcome unrealistic edge effects observed with the use of simplified models (Kerr, 1964; Kerr \& Coffin, 1991; Reissner, 1958; Vlassov, 1966). Some other studies have been devoted to the time evolution of the foundation response (Omurtag \& Kadıoglu, 1998) often focus on the structural response (Kneifati, 1985; Sironic, Murray, \& Grzebieta, 1999) or to stability studies of columns on foundations of various kind (Lin \& Adams, 1987). The Hetenyi (1950) model is also a two-parameter elastic model. Kerr (1984) outlines the presentation and comparison of the elastic models.

Furthermore, Di Paola, Marino, \& Zingales (2009) showed that elastic foundation models might be referred to as gradient models, which involve some nonlocality. By the variation formulation of the Reissner model, Challamel, Meftah, \& Bernard (2010) investigated the buckling of an axially loaded Euler-Bernoulli model supported on an elastic nonlocal medium. Nobili (2012) presents the Hamiltonian variation formulation of the contact problem for a Euler-Bernoulli beam on Pasternak foundation. Horvath \& Colasanti (2011) investigate another one subgrade model with spring coupling called the modified Kerr-Reissner model. Worku (2014) develops Pasternak or Filonenko-Borodich models. The simplest continuum model of Vlahos (1966) is compared and parameters evaluated.

In this paper, the Filonenko-Borodich model of the spring system under the membrane is investigated. The force at the point and the continuous uniform load are applied. A novel procedure to add the 
perfect gas pressure between the membrane and the rigid foundation is proposed. Modification of the solution and a significant increase in accuracy is investigated.

\section{Concentrated normal force}

An elastic half-space is loaded one-dimensionally over a narrow strip (the line loading), a state of plane strain is assumed. The concentrated force of intensity $P$ per unit length, distributed along the $y$-axis, causes a surface displacement (Johnson, 1987):

$$
w_{J}=\frac{2 P}{\pi E^{*}} \log \frac{x_{o}}{x}
$$

where $E^{*}=\frac{E}{1-v^{2}}$ and $E$ is Young modulus, MPa; $v$ - Poisson ratio (Figure 1). When $x \rightarrow \infty$ the displacements $w_{J} \rightarrow-\infty$ if elastic half-space has no limits. The distance $x_{0}$, where displacements $w_{J}=0$, depends on the real sizes of the elastic body $l$ and $h$. The Winkler model is unsuitable for the concentrated force, but when a membrane is placed over the distributed spring system, the displacements $w_{m}$ are bounded on the whole surface $-\infty<x<+\infty$.

Equilibrium of the membrane yields is:

$$
\begin{gathered}
T \frac{d^{2} w}{d x^{2}}=k w, \\
F_{z}=-k w,
\end{gathered}
$$

Where $T$ - the tension force per unit length, $\mathrm{N} / \mathrm{m} ; F_{z}-$ the spring force component, $\mathrm{N} / \mathrm{m}^{2}$.

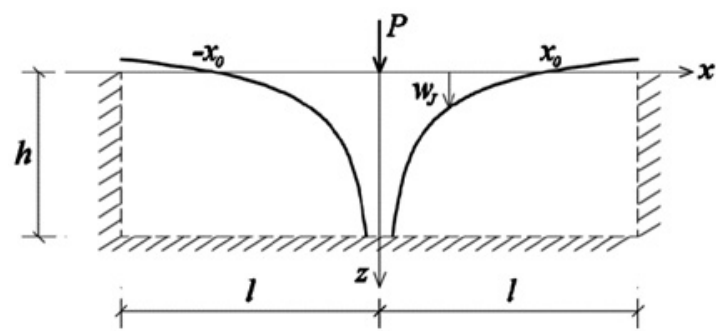

Note: the concentrated force $P$ is distributed along the $y$-axis.

Figure 1. Displacements of an elastic body surface 
The solution to the Eq. (2) is

$$
w=C_{1} e^{-r x}+C_{2} e^{r x}, r=\sqrt{\frac{k}{T}}
$$

bounded when $C_{2}=0$. The total force applied to the whole membrane when $x \geq 0$ is

$$
\sum F_{z}=\frac{P}{2}-k \int_{0}^{\infty} w d x=0 .
$$

The constant $C_{1}=\frac{r P}{2 k}$ and solution Eq. (4) is

$$
w_{m}=\frac{r P}{2 k} e^{-r x} .
$$

Let deflection line Eq. (6) of the membrane has to be approximated to the solution Eq. (1) inside the interval $\left[-x_{v}+x_{v}\right]$. If $\xi=\frac{x}{x_{v}}, \xi=\frac{x_{0}}{x_{v}}$ then the difference $w_{J}-w_{m}=\frac{2 P}{\pi E^{*}} \delta(\xi)$, where

$$
\delta(\xi)=\log \frac{\xi_{0}}{\xi}-S_{m} e^{-0 \xi}
$$

and

$$
S_{m}=\frac{\pi E^{*}}{4 \sqrt{k T}}, \mathrm{v}=r x_{v}=x_{v} \sqrt{\frac{k}{T}} .
$$

The constants $S_{m}$ and v, which minimise the integral (Eq. (9))

$$
\Gamma\left(S_{m}, v\right)=\int_{0}^{1} \delta^{2}(\xi) \delta \xi
$$

need to be estimated. The tension force and the spring constant follows from Eq. (8) $T=\frac{\pi x_{v} E^{*}}{4 v S_{m}}$, and $k=\frac{\pi v E^{*}}{4 x_{v} S_{m}}$. Does the problem be what real possibilities of approximation of the logarithm function by exponentials are? The ratio $\xi_{0}=\frac{x_{0}}{x_{v}}$ in Eq. (7) is specified by the elastic body.

The integral Eq. (9) is

$$
\Gamma=\left(2+\log \xi_{0}\right) \log \xi_{0}+2-2 S_{m} \frac{1-e^{-\mathrm{v}}}{\mathrm{v}}+S_{m}^{2} \frac{1-e^{-2 \mathrm{v}}}{2 \mathrm{v}}-2 S_{m} \phi(\mathrm{v})>0
$$

where the function $\phi(\alpha)=\int_{0}^{1} e^{-\alpha \xi} \log \xi d \xi=\frac{1}{\alpha} \int_{0}^{1} \frac{e^{-\alpha \xi}-1}{\xi} d \xi$. This function presented by the series

$$
\phi(\alpha)=\sum_{j=1}^{\infty} \frac{(-\alpha)^{j-1}}{j \cdot j !}
$$


convergent for all $|\alpha|<\infty$. Nevertheless, if $|\alpha|>4.7$ it is better to apply asymptotic approximation (Prudnikov, 2018):

$$
\Phi(\alpha)=\frac{\varepsilon^{-\alpha}}{\alpha^{2}}\left[\sum_{j=0}^{\infty} \frac{j !}{(-\alpha)^{j}}\right]+\frac{\log \alpha+C_{e}}{\alpha},
$$

Elastic Foundation

which is deduced substituting exponential integral $E_{i}(x)=\int_{-\infty}^{x}\left(\frac{e^{x}}{x}\right) d x$, $C_{e}=0.577216$ is the Euler constant. If the terms with $j>14$. (Eq. (11)) and with $j>9$ (Eq. (12)) are neglected, the error for both series $<0.00009 \%$ or $0.023 \%$ as $\Phi(4.7)=0.45246$.

From the Eq. $\frac{\partial \Gamma}{\partial S_{m}}=0$, the solution for $S_{m}$, like a function of $v$, is deduced, and then the function $\Gamma=\Gamma\left(S_{m}(v), v\right)$ depends only on $v$.

In Figure 2, the deflections $w_{J}$ and $w_{m}$ are displayed when the interval of interest is $\left[-x_{v},+x_{v}\right], x_{v}=2 x_{0}$ When $x \rightarrow \infty$ the membrane displacements Eq. (6) are positive, but displacements of the elastic halfspace surface line, if $x=x_{v}$, are negative. Therefore the relative error $\phi=\sqrt{\frac{\int_{0}^{1} \delta^{2}(\xi) \delta \xi}{\int_{0}^{1} \delta_{m}^{2}(\xi) \delta \xi}} \cdot 100 \%$ of the best approximation $\left(S_{m}=2.40\right.$, when $v=1.004$ ) is $\phi \approx 58.8 \%, \delta_{m}=S_{m} e^{-v \xi}$. If $\xi_{0}=1, x_{v}=x_{0}, v=1.004, S_{m}=3.50$ and relative error $\phi=17.3 \%$. When the concentrated normal force is applied,

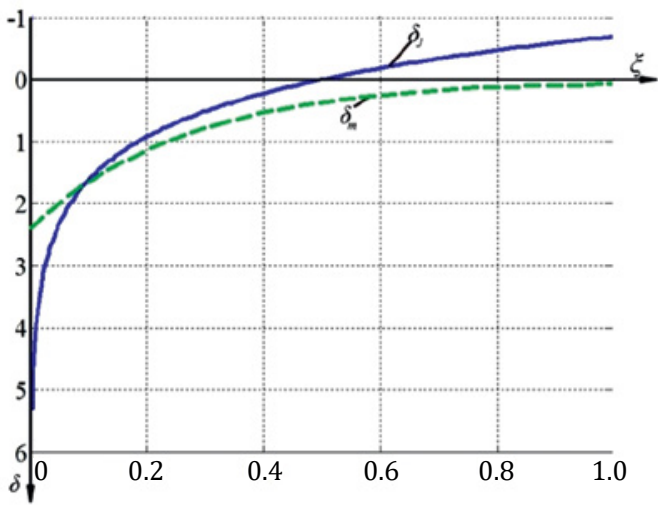

Figure 2. Deflections $\delta_{J}$ and $\delta_{m}$ as functions of the relative distance $\xi$ when $\xi_{0}=0.5$ and concentrated force $P$ is applied 
deflections of the elastic body $w_{J} \rightarrow \infty$ if $x \rightarrow 0$, whereas deflection of the membrane at $x=0$ is $w_{m}=\frac{r P}{2 k}=\frac{2 S_{m} P}{\pi E^{*}}, \delta_{m}=\frac{\pi E^{*} w_{m}}{2 P}=S_{m}$; consequently, a substantial error is caused by the principle presumptions of these two models.

\section{Ideal gas under the membrane}

A significant correction in the membrane model is achieved if an ideal gas is added under the membrane (Figure 3). The pressure of the gas is assumed $q_{0}$ when the force $P=0$ and the pressure is $q_{0}+q$, if $P$ is applied. The resultant force component now is $F_{z}=-k w-q$, and solution Eq. (6) is $w_{m}=\frac{r P}{2 k} e^{-r x}-\frac{q}{k}$. Equilibrium condition Eq. (5) is $\sum F_{z}=\frac{P}{2}-k \int_{0}^{l} w d x-q l=0$, where $l$ is the horizontal size of the body and the membrane shown in Figures 1 and 3. It is assumed that $l$ is sufficiently large and $e^{-r l} \approx 0$. If the temperature of the gas is constant, Boyle law is given by $q_{0} V_{0}=\left(q_{0}+q\right) V$, where $V_{0}=h_{0} l, V=\int_{0}^{l}\left(h_{0}-w_{m}\right) d x=h_{0} l-\frac{P}{2 k}+\frac{q}{k} l$. Therefore the Eq. of state $\left(1+\frac{q}{q_{0}}\right)\left(1-\frac{P}{2 k_{0} l}+\frac{q}{k h_{0}}\right)=1$ is non-linear concerning $q$, but if $|q|<<q_{0}$ it is approximated as $q\left(1+\frac{k h_{0}}{q_{0}}\right)=\frac{P}{2 l}$. The difference function

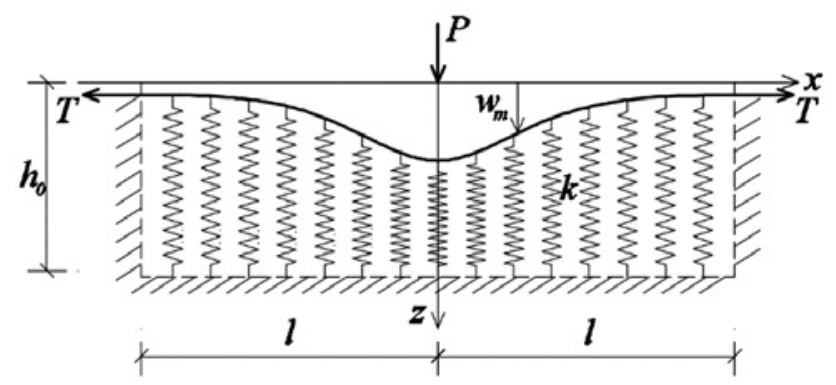

Figure 3. Displacements of a membrane over the spring system when concentrated force is distributed along the $y$-axis 


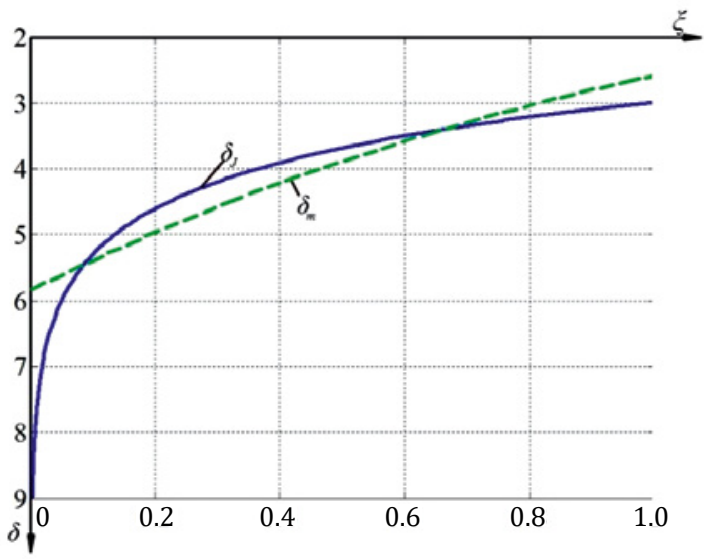

Figure 4. Deflections $\delta_{J}$ and $\delta_{m}$ as functions of $\xi$ when $\xi_{0}=20$ and concentrated force $F$ is applied.

Eq. (7) now is

$$
\delta=\log \frac{\xi_{0}}{\xi}-S_{m} e^{-v \xi}+S_{0}
$$

where

$$
S_{0}=\frac{\pi E^{*}}{4 k l} \cdot \frac{1}{1+\frac{k h_{0}}{q_{0}}}
$$

The dimensionless number $S_{0}=\log g_{0}$ in Figure 3 is positive, but if the cavity for the ideal gas is above the membrane, the volume of $S_{0}$ is negative. Eq. (13) reduced to $\delta=\log \frac{\xi_{0} g_{0}}{\xi}-S_{m} e^{-v \xi}, 0<g_{0}<\infty$. Selecting the value of $\frac{h_{0}}{q_{0}}$ in Eq. (14), vary the product $\xi_{0} g_{0}=\xi_{0}^{*}$. Mostly the value of $\xi_{0}^{*}$ for which error of membrane approximation is minimal, then calculate the constants $g_{0}=\frac{\xi_{0}^{*}}{\xi_{0}}=\exp \left(S_{0}\right)$ and $\frac{h_{0}}{q_{0}}$ is calculated. If the gas pressure is included, the displacements $w_{m}$ are negative for $x>x_{0}$, as it is for the elastic bodies (Figure 1).

When concentrated normal force is applied, $\xi_{0}^{*}=20$ is determined and then parameters $v=1.004, S_{m}=5.834$ calculated. The integral Eq. (9) for these values $\Gamma=0.188$ and a relative error is only $\phi \approx 9.9 \%$ (Figure 4). 


\section{Distributed load}

The action of a distributed load generates moderate surface deflection line of the elastic body (Johnson, 1987) $w_{J}=\frac{p_{0}}{\pi E^{*}}\left[\begin{array}{c}a C_{s}-(a+x) \log (a+x)^{2}- \\ (a-x) \log (a-x)^{2}\end{array}\right]$, where $p_{0}$ is load per square unit, $2 a$ - width of the load belt (Figure 5), $C_{s}$ - the constant, similar to $\log x_{0}$ in Eq. (1). The constant $C_{s}$ depends on the sizes $l$ and $h$ of the elastic body.

The differential Eq. of the membrane equilibrium is

$$
\left\{\begin{array}{c}
T \frac{d^{2} w}{d x^{2}}+p=k w+q, \quad|x| \leq a \\
T \frac{d^{2} w}{d x^{2}}=k w+q, \quad|x| \geq a
\end{array}\right.
$$

A solution to this Eq.

$$
\left\{\begin{array}{cc}
\frac{k}{p} w_{m}(x)=1-e^{-r a} \cosh (r x)-\frac{q}{p}, & 0 \leq x \leq a, \\
\frac{k}{p} w_{m}(x)=\sin h(r a) e^{-r x}-\frac{q}{p}, & a \leq x .
\end{array}\right.
$$

The only fixed interval $[-a,+a]$ for the approximation of the lines $w_{J}$ and $w_{m}$ is investigated. If $w_{J}-w_{m}=\frac{p_{0} a}{\pi E^{*}} \delta(\xi)$, where $\xi=\frac{x}{a}$, then

$$
\begin{gathered}
\delta(\xi)=S_{0}-(1+\xi) \log (1+\xi)^{2}-(1-\xi) \log (1-\xi)^{2}- \\
-S_{m}\left(1-e^{-v} \cosh (v \xi)\right)
\end{gathered}
$$

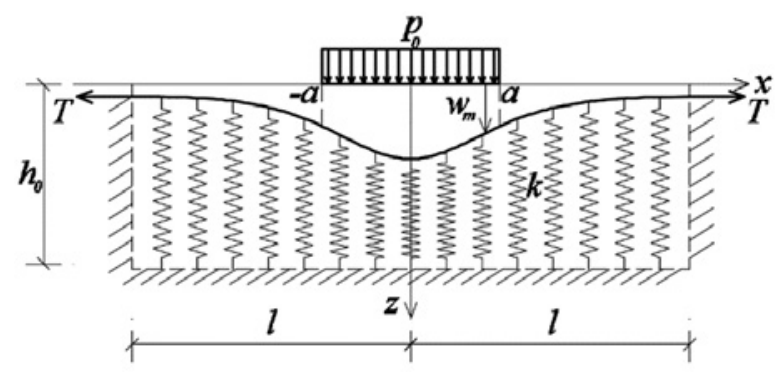

Figure 5. Displacements of a membrane over the spring system for the uniform distributed load 


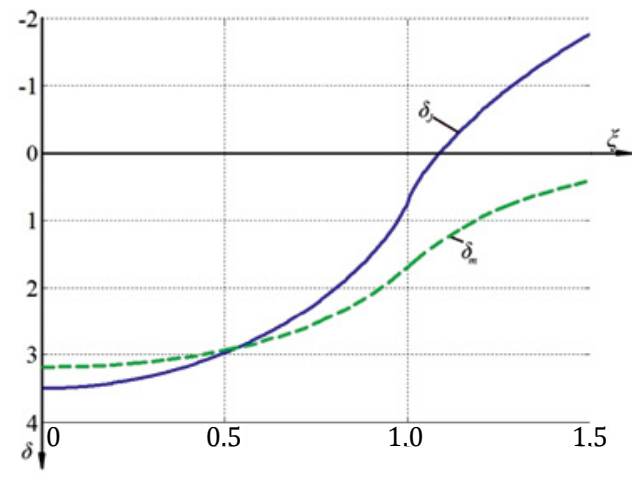

Figure 6. Deflections $\delta_{J}$ and $\delta_{m}$ as functions of $\xi$ when $S_{S}=3.5$ for the uniform distributed load

In Eq. (16) $S_{0}=C_{s}-\frac{\pi E^{*}}{k a} \frac{q}{p_{0}}, S_{m}=\frac{\pi E^{*}}{k a}, v=r a$. The integral Eq. (9) is deduced applying Eq. (15). The constants $S_{m}(v)$ and $v$ that minimise the integral Eq. (9) then are found. If $C_{S}=S_{0}=3.5$ the values $v=2.802$, $S_{m_{\text {min }}}=3.378$, the relative error $\phi \approx 12.0 \%$. The constant $C_{S}(l, h)$ is dependent on the size of the elastic body. The values of $\delta_{J}=\frac{p_{0} a}{\pi E^{*}} w_{J}, \delta_{m}=\frac{p_{0} a}{\pi E^{*}} w_{m}$ are displayed in Figure 6 . When $\xi$ increases, the line $\delta_{J}(\xi)$ intersects the line $\delta_{J}=0$ and becomes negative, while the $w_{m}$ is positive for all $\xi$ and approaches zero when $\xi \rightarrow \infty$ Eq. (15).

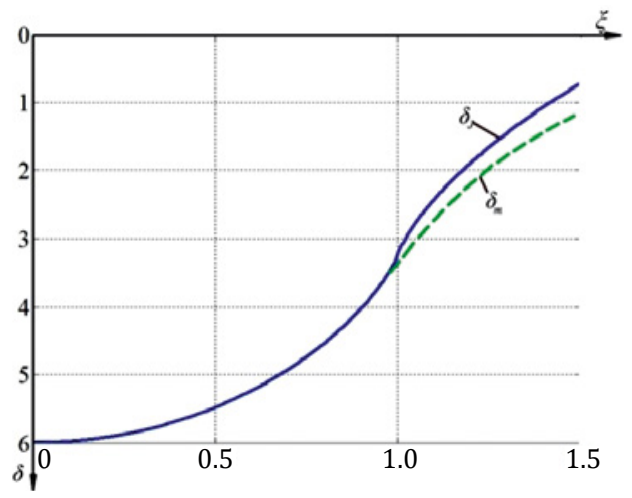

Figure 7. Deflections $\delta_{J}$ and $\delta_{m}$ as functions of $\xi$ when $S_{S}=6.0$ for uniform distributed load and ideal gas pressure 
In Figure 7, the displacements are displayed when $S_{0}=6.0$. If $C_{s}=3.5$, the negative ideal gas pressure have to be added:

$$
\frac{q}{p_{0}} \frac{\pi E^{*}}{k a}=C_{s}-S_{o} .
$$

Parameters of the membrane are $v_{\min }=2.13, S_{m_{\min }}=6.80$ and relative error $\phi \approx 0.31 \%$. Relative error is minimal because of large values of the displacement $\delta_{m}$.

\section{Conclusions}

1. Solving elastic continuum equations presents well-known mathematical problems. A solution of the Filonenko-Borodich model with a membrane or the Pasternak model with shear interaction between the spring elements is relatively simple. The accuracy of the solution is significantly increased by adding an ideal gas pressure, linearly dependent on the external load.

2. A more complicated and most likely better solution has the Hettenyi or Reissner models. Additional pressure can be used for these models.

3. Adding the ideal gas pressure improves the accuracy of the approximate solution for all elastic foundation models.

\section{REFERENCES}

Ashley, H. (1956). Piston theory-a new aerodynamic tool for the aeroelastician. Journal of the Aeronautical Sciences, 23(12), 1109-1118. https://doi.org/10.2514/8.3740

Challamel, N., Meftah, S. A., \& Bernard, F. (2010). Buckling of elastic beams on non-local foundation: A revisiting of Reissner model. Mechanics Research Communications, 37(5), 472-475. https://doi.org/10.1016/j.mechrescom.2010.05.007

Di Paola, M., Marino, F., \& Zingales, M. (2009). A generalized model of elastic foundation based on long-range interactions: Integral and fractional model. International Journal of Solids and Structures, 46(17), 3124-3137. https://doi.org/10.1016/j.ijsolstr.2009.03.024

Filonenko-Borodich, M. M. (1940). Some approximate theories of elastic foundation. Uchenyie Zapiski Moskovkogo Gosudarstuennogo Universiteta Mekhanika, Moscow, 46, 3-18.

Hetenyi, M. (1950). A general solution for the bending of beams on an elastic foundation of arbitrary continuity. Journal of Applied Physics, 21(1), 55-58. https://doi.org/10.1063/1.1699420 
Horvath, J. S., \& Colasanti, R. J. (2011). Practical subgrade model for improved soil-structure interaction analysis: model development. International Journal of Geomechanics, 11(1), 59-64. https://doi.org/10.1061/(ASCE)GM.1943-5622.0000070

Johnson, K. L. (1987). Contact mechanics. Cambridge university press.

Kerr, A. D. (1964). Elastic and viscoelastic foundation models. Journal of Applied Mechanics, 31(3), 491-498. https://doi.org/10.1115/1.3629667

Kerr, A. D. (1984). On the formal development of elastic foundation models. Ingenieur-Archiv, 54(6), 455-464. https://doi.org/10.1007/BF00537376

Kerr, A. D., \& Coffin, D. W. (1991). Beams on a two-dimensional Pasternak base subjected to loads that cause lift-off. International Journal of Solids and Structures, 28(4), 413-422. https://doi.org/10.1016/0020-7683(91)90057-M

Kneifati, M. C. (1985). Analysis of plates on a Kerr foundation model. Journal of Engineering Mechanics, 111(11), 1325-1342. https://doi.org/10.1061/(ASCE)0733-9399(1985)111:11(1325)

Lin, L., \& Adams, G. G. (1987). Beam on tensionless elastic foundation. Journal of Engineering Mechanics, 113(4), 542-553. https://doi.org/10.1061/(ASCE)0733-9399(1987)113:4(542)

Nobili, A. (2012). Variational approach to beams resting on two-parameter tensionless elastic foundations. Journal of Applied Mechanics, 79(2), 021010. https://doi.org/10.1115/1.4005549

Omurtag, M. H., \& Kadıoğlu, F. (1998). Free vibration analysis of orthotropic plates resting on Pasternak foundation by mixed finite element formulation. Computers \& structures, 67(4), 253-265. https://doi.org/10.1016/S0045-7949(97)00128-4

Pasternak, P.L. (1954). On a new Method of Analysis of an Elastic Foundation by Means of Two Foundation Constants. Gosuderevstvennae Izdatlesva Literaturi po Stroitelstvu i Arkihitekture, Moscow, USSR (in Russian)

Prudnikov, A. B. (2018). Integrals and series. Routledge.

Reissner, E. (1958). A note on deflections of plates on a viscoelastic foundation. J. Appl. Mech., ASME, 25, 144-145.

Sironic, L., Murray, N. W., \& Grzebieta, R. H. (1999). Buckling of wide struts/ plates resting on isotropic foundations. Thin-walled structures, 35(3), 153-166. https://doi.org/10.1016/S0263-8231(99)00029-4

Vasani, P. C. (2003). Interactive analysis models for soil and structures. Structural Engineering Foru of India.

Vlasov, V. Z. (1966). Beams, plates and shells on elastic foundation. Israel Program for Scientific Translation.

Winkler, E. (1867). Die Lehre von der Elastizität und Festigkeit. Dominicus, Prague.

Worku, A. (2014). Development of a calibrated Pasternak foundation model for practical use. International Journal of Geotechnical Engineering, 8(1), 26-33. https://doi.org/10.1179/1938636213Z.00000000055 